\title{
Saisir les identités en mouvement : parenté et histoires de familles turques en migration
}

Grasping Identities on the Hove: Parenthood and Histories of Turkish Migrant Families

Captar las identidades en movimiento: parentesco e historias de familias turcas en la migración

\section{Claire Autant-Dorier}

\section{OpenEdition}

\section{Journals}

Édition électronique

URL : https://journals.openedition.org/remi/4995

DOI : $10.4000 /$ remi.4995

ISSN : $1777-5418$

Éditeur

Université de Poitiers

Édition imprimée

Date de publication : 1 décembre 2009

Pagination : 133-151

ISBN : 978-2-911627-53-8

ISSN : 0765-0752

\section{Référence électronique}

Claire Autant-Dorier, «Saisir les identités en mouvement : parenté et histoires de familles turques en migration », Revue européenne des migrations internationales [En ligne], vol. 25 - n³ | 2009, mis en ligne le 01 décembre 2012, consulté le 16 avril 2022. URL : http://journals.openedition.org/remi/4995 ; DOI : https://doi.org/10.4000/remi.4995 


\section{Saisir les identités en mouvement : parenté et histoires de familles turques en migration}

\section{Claire AUTANT-DORIER*}

Nous proposons de revisiter ici un parcours de recherche mené auprès de populations turques pendant plusieurs années afin d'interroger en quoi pourrait consister une approche anthropologique de l'immigration. De l'analyse de la parenté, classique s'il en est de l'anthropologie, à l'analyse des identités en mouvement, nous montrerons comment la perspective adoptée conduit à privilégier une anthropologie de la mobilité au delà du seul phénomène de l'immigration et des questions qu'elle pose à la société d'accueil. Il ne s'agira toutefois pas de défendre ici une anthropologie close sur elle même qui pourrait mieux que d'autres prétendre rendre compte des phénomènes migratoires. Notre démarche a, au contraire, constamment été alimentée d'apports et de réflexions de disciplines voisines, la sociologie en premier lieu mais aussi la géographie ${ }^{1}$. Mais nous verrons comment se traduit concrètement, dans la démarche de terrain comme dans celle d'écriture, ce qui pourrait relever d'une spécificité de l'anthropologie. Nous mettrons à l'épreuve quelques outils et ressources théoriques susceptibles de saisir ce que nous proposons d'appeler des identités en mouvement.

\section{CHEMINER AVEC LES MIGRANTS}

\section{Une perspective anthropologique pour éviter l'écueil de l'immigration comme problème social}

Notre première approche du terrain se donnait comme principe assez classique de comprendre comment les immigrés prennent place dans l'espace d'installation en

\footnotetext{
* Mcf, université Jean Monnet Saint Etienne, Chercheure au MoDyS (UMR 5264), 6 rue Basse des Rives, 42023 Saint Etienne Cedex 2 ; claire.autant.dorier@univ-st-etienne.fr

1 Jean et Françoise Métral au GREMMO (dans la Maison de L'Orient à Lyon largement pluridisciplinaire) ont notamment été des artisans formidables de séminaires croisant anthropologie de la parenté et ethnologie urbaine, histoire et sociologie, géographie physique et humaine. Les collaborations avec Alain Battegay ont également entretenu cette perspective, comme les échanges avec Isabelle Rigoni et Stéphane de Tapia sur les migrations de Turquie.
} 
France : il s'agissait d'étudier comment une « communauté » turque prenait place dans une commune rurale de taille moyenne (5 000 habitants). Le principe de la monographie localisée rassemblait, pour l'apprentie anthropologue que nous étions, à la fois l'imaginaire ethnographique de la tribu isolée dans laquelle on s'immerge, et celui du quartier village cher à l'anthropologie urbaine. Ces représentations furent toutefois rapidement mises à mal, tant sur l'idée d'une communauté homogène que sur celle d'un lieu unique comme espace de significations. « (...) peu à peu, s'est imposée une parole : une vision du monde, une manière collective et originale de la dire et de l'expliquer. » (Zonabend, 1980 : 6). Au « parler famille », qu'évoque ici Françoise Zonabend se combine ce qu'on pourrait appeler le « parler nomade ", c'est-à-dire la vision du monde particulière que construit la mobilité, telle que la décrivent Philippe Videlier (Videlier, 1993) ou Arjun Appaduraï $(2001)^{2}$. Ces récits m'ont ainsi orientée d'une part vers la Turquie, mais pas n'importe où en Turquie - là où des membres de la parenté se trouvent et se retrouvent — et d'autre part vers les alliances - temps particulièrement fort où se réactivent et se construisent les liens de parenté, recréant du lien là où les distances géographiques le menacent. La question « incontournable » de l'intégration est apparue dès lors comme singulièrement inadéquate!

L'importance du groupe de parenté comme appui ou comme référence dans l'orientation des choix matrimoniaux a fait l'objet d'une seconde étape de recherche. Si les outils de l'anthropologie de la parenté classique (c'est-à-dire plutôt structuraliste) ont eu une utilité pour nous dans l'étude des migrations, ce fut pour sortir d'une vision ethnocentrique et assimilationniste qui tend à considérer les pratiques matrimoniales des immigrés comme des traditions liberticides appelées à disparaître. Cela permettait de considérer les mariages dans la parenté, arrangés par les parents, non pas comme des mariages « forcés » « consanguins », mais d'abord comme des manifestations de formes d'alliance bien identifiées ; notamment le mariage avec la cousine croisée matrilatérale caractérisant l'échange généralisé décrit par C. Lévi-Strauss (Lévi-Strauss, 1967). En revanche, comprendre la parenté en situation migratoire imposait de ne pas s'en tenir aux classifications formelles mais d'en comprendre les usages dans une perspective plus bourdieusienne (Bourdieu, 1980).

Au-delà du domaine de l'alliance, notre hypothèse de recherche a été de considérer que la parenté, comprise comme l'ensemble des relations généalogiques dans lesquelles un individu s'inscrit, constitue un facteur essentiel des phénomènes migratoires et dans le même temps une perspective d'analyse heuristique pour le chercheur qui tente de rendre compte de ceux-ci. Alors que l'on considère souvent, dans les travaux sur les phénomènes migratoires, les caractéristiques culturelles de la parenté comme des facteurs de résistance au changement, nous avons fait l'hypothèse que la parenté accompagne et permet des changements, elle en est aussi la caisse de résonance.

2 Ils montrent comment des individus inscrits dans des parcours et expériences migratoires de type diasporique ont une conception des proximités et des distances qui subvertit les ordres de la sédentarité : faisant du point le plus lointain et exotique un espace de référence familier pour peu que l'on soit passé par là ou que «l'un des siens » s'y trouve. 


\section{Du mésusage de l'anthropologie}

Cette hypothèse de l'importance des formes de la parenté dans les formes de la migration a été mise en œuvre de façon discutable par Emmanuel Todd (Todd, 1994). Qu'il nous soit permis, en contre point de la présentation de notre démarche, de dénoncer l'usage qu'il fait de l'anthropologie (de la parenté) pour analyser l'immigration. Emmanuel Todd propose de rendre compte des modalités d'assimilation des populations migrantes dans divers pays occidentaux. Pour lui, les structures fondamentales de la parenté (schématisées comme différentialistes ou égalitaristes) qui caractérisent les sociétés de résidence et les sociétés de provenance, permettraient d'expliquer les tendances à l'assimilation ou à la ségrégation constatées dans les démocraties occidentales considérées. Ainsi évoquet-il l'idée d'un " destin des immigrés » selon leur pays de résidence, prédéfini par ces structures fondamentales dont il fait la clé unique d'analyse. «Les structures familiales apparaissent ici fondatrices, à travers les représentations qui en découlent : là où l'on pense les frères différents, on ne peut échapper à la vision d'une humanité diversifiée et segmentée » (Todd, 1994 :13). Peu importe que l'on ne puisse vérifier si ces schémas se transmettent toujours dans la famille contemporaine, l'inconscient du peuple continuerait de s'exprimer. Ainsi, par exemple, le système idéologique plutôt égalitariste des ÉtatsUnis ne parviendrait pas à décoller de sa matrice anthropologique fondamentalement différencialiste, ce qui explique le maintien de la ségrégation des Noirs.

En dehors des écueils évidents de cette comparaison à multiples échelles géographiques et temporelles, il se trouve que le cas des Turcs en France ne peut être expliqué avec ce modèle pourtant séduisant. Les approximations et contradictions qui jalonnent sa démonstration sur le cas turc sont tout à fait efficaces pour remettre en cause ses présupposés. Rappelons les deux postulats posés comme points de départ de l'ensemble de la démonstration : premièrement, les structures fondamentales de la parenté n'ont pas évolué dans le temps long et elles détermineraient la façon dont une société envisage son rapport à l'Autre. Deuxièmement, les logiques du pays d'accueil l'emporteraient dans la relation. Or, les Turcs (structure égalitariste) auraient réussi l'exploit d'adopter les logiques structurelles du système Allemand (différentialiste) et à les réimporter en France (structure à dominante égalitariste ${ }^{3}$ ). Démonstration pour le moins surprenante, qui oublie de plus que les Turcs de France ne sont pas passés par l'Allemagne. Ils auraient donc réussi cet emprunt, contraire aux structures fondamentales de leur système de parenté, par « diffusion »!

Ce parti-pris conduit à évaluer, à la manière d'un jugement définitif, les capacités ou les possibilités d'assimilation des populations immigrées dans une perspective anhistorique et essentialiste. L'idée de destin, symptomatique de cette perspective (même si elle est invoquée parfois par les migrants eux-mêmes), présente toutes les séductions d'une explication définitive. Elle en a tous les défauts lorsqu'elle prétend être scientifique. Car

3 Le cas français, sans doute parce qu'il est mieux connu des lecteurs, ou parce que nous sommes un peuple plus subtil (?), est plus nuancé que celui des autres pays. E. Todd distingue en fonction des régions des systèmes différencialistes et égalitaristes, mais en revanche ne prend pas en compte les formes et usages contemporains de la famille. Au contraire les particularités du système de parenté turc (qui combinent des principes de la parenté issus de l'ère asiatique et ceux de l'aire arabo-musulmane) sont négligées. 
elle masque justement le processus de « mise en intrigue » (Ricœur, 1983) qui amène à conclure sur l'orientation de ce destin. Double processus de mise en scène de soi par le migrant en interaction avec son environnement, et de construction et de présentation des résultats par le chercheur qui doit être interrogé en soi.

De façon plus générale les travaux sociologiques sur l'immigration ont tendance à réduire la question de la famille au conflit entre générations, ou au problème du statut de la femme et à faire du mariage mixte un indicateur immédiat d'assimilation. L'étude des alliances dans ce contexte est souvent menée indépendamment d'une analyse des parcours familiaux, en considérant trop souvent des individus atomisés. Il semblait au contraire nécessaire de considérer les dynamiques qui traversent le champ familial dans leur ensemble, et de ne pas isoler les personnes et les cellules familiales des réseaux dans lesquels elles sont engagées. Paradoxalement, alors que les premières recherches sur l'immigration turque avaient souligné l'importance des réseaux de parenté comme base des réseaux régionaux et ethniques de solidarité, il n'y a guère eu de véritables recherches orientées sur le caractère familial de cette migration ${ }^{4}$. C'est à décrypter ce qui se joue dans les réseaux de parenté en migration que nous nous sommes donc attachée.

\section{Intérêts et limites d'une ethnographie multilocalisée et du suivi des parcours dans la durée}

L'enquête a ainsi porté sur neuf groupes familiaux installés dans le département de la Loire, dans divers communes, et originaires de plusieurs régions en Turquie. La diversité des configurations familiales et des contextes d'installation devait permettre de mieux rendre compte de la différenciation des carrières des migrants ${ }^{5}$. Nous avons suivi les trajectoires des migrants installés en France pendant 5 à 8 ans lors de visites régulières en France et en Turquie (Autant-Dorier, 2007). Nous avons réalisé auprès de ces groupes familiaux des entretiens individuels ou collectifs de type récit de vie ; un travail de reconstitution de généalogie et de collecte des histoires de famille a permis de situer les positions et parcours des autres membres de la parenté dont nous avons rencontré certains. Le travail de (re)-construction généalogique constitue, en même temps qu'un mode de collecte de données, un révélateur des enjeux de recompositions et repositionnement au sein de la parenté et des réseaux villageois dans le contexte migratoire. La mémoire des relations et la lecture qui en est faite donne à voir les rôles et la position de celui qui les restitue : logique lignagère plus masculine, logique pratique plus féminine, perspective historique partant d'un ancêtre ou point de vue construit à partir de soi et des évènements vécus.

4 Le volet « famille » de la recherche dirigée par Altan Gökalp pour le FAS constituait une exception (Gökalp, Kastoryano et de Tapia, 1997). La thèse de Benoît Fliche (2007) a depuis examiné certains aspects des usages de la parenté en migration.

5 Dans certains cas seule une cellule familiale est installée en France, et nous avons rencontré d'autres membres de la parenté en Turquie. Dans deux cas les familles installées en France comportaient plusieurs cellules familiales, les primo-migrants étant venus en France à plusieurs frères ou père et fils (ce qui représente environ 60 à 70 personnes installées souvent à proximité immédiate). Les configurations repérées ne se limitaient pas au réseau de parenté mais identifiaient également l'importance de la présence d'amis et de compatriotes issus du même village, ou ayant connu les mêmes étapes migratoires. 
Comprendre les logiques des migrants supposait de prendre conjointement en considération l'ici et l'ailleurs (on devrait dire les ici et les ailleurs). C'est à dire analyser les transformations du groupe migrant et considérer ce qui se passe dans le milieu d'arrivée, comme dans le milieu de départ. Nous rejoignons ainsi les propositions de Jean-Noël Ferrié et Gilles Boetsch (1992 : 7-8) concernant une anthropologie de l'immigration, même s'il semble préférable de parler d'anthropologie de la migration : « Les anthropologues, naguère charmés par l'exotisme tropical puis par l'exotisme bucolique des campagnes européennes, se sont aperçu que le monde urbain de leur vie quotidienne recelait suffisamment d'étrangeté pour les satisfaire. L'interprétation pessimiste de cette évolution étant que l'on atteignait la fin de la discipline ou une transformation de l'anthropologie en microsociologie. Dès lors il est néanmoins tentant de présenter l'anthropologie de l'immigration comme une subdivision de l'anthropologie urbaine, ce qui serait sans doute conforme au point de vue de l'École de Chicago. (...) Toutefois si l'anthropologie urbaine s'intéresse aux groupes immigrés comme une des composantes de la ville, l'anthropologie de l'immigration étudie elle, la dynamique propre de ces groupes. Ce qui veut dire : (a) la transformation du groupe migrant entre le milieu de départ et le milieu d'arrivée ; (b) l'effet sur le milieu d'arrivée ; (c) l'effet sur le milieu de départ. »

Notre démarche méthodologique, et c'est en cela aussi que nous la définirions comme anthropologique, tente de situer à proximité de l'expérience ordinaire des migrants, de leurs récits, des significations que prennent pour eux les espaces qu'ils fréquentent, les sens qu'ils donnent à leurs activités. L'ethnographie multilocalisée des lieux et des relations permet d'en saisir les imbrications et les variations. Le parti-pris d'accompagner les populations étudiées dans le temps et dans l'espace permet de mesurer les épreuves et situations qu'elles ont à affronter comme les ressources et cadres sur lesquels elles s'appuient. Nous notons cependant (Autant-Dorier et Battegay, 2007) que l'investissement personnel et le temps qu'implique ce type de démarche conduisent nécessairement à limiter la taille des unités ainsi abordées. Les « populations » qui font l'objet de ce type d'investigation, ou du moins qui sont au cœur de celle-ci, sont souvent réduites à quelques individus ou à de petits groupes ${ }^{6}$. De même les parcours appréhendés sont davantage des fragments ou des moments de parcours que des itinéraires saisis dans leur totalité. Enfin, les situations et interactions saisies demeurent relativement ponctuelles et les observations non systématisables. Ainsi, malgré la richesse des observations et recueils effectués, se pose la question des interprétations que l'on en fait. Le risque est bien réel, et attesté par nos expériences, de se laisser aller à des prédictions, dans le meilleur des cas à des hypothèses, qui sont pour le moins fragiles. Les basculements, réorientations, recompositions auxquels nous avons eu à faire dans le suivi des familles turques montrent la labilité des situations et des interprétations qui peuvent en être faites. Se pose alors une double question : celle des modes de restitution et celle des outils conceptuels à même de rendre compte de cette labilité.

6 La démarche discutée ici s'inscrit dans un ensemble de travaux qui avaient été conduits dans le cadre de la Maison de l'Orient (GREMMO) et participe plus largement de recherches développées autour des mobilités et des ressources des migrants, notamment par Alain Tarrius. 


\section{DÉCRIRE LA MOBILITÉ : DES RÉSEAUXAUX ATTACHEMENTS}

C'est à travers certains épisodes de l'histoire singulière d'une famille que nous tenterons de montrer comment, à la difficulté d'appréhender une réalité en mouvement, se mêle le souci du choix des termes pour en rendre compte. Progressivement l'appareillage conceptuel s'est trouvé pris en défaut : l'analyse du sens pratique et des habitus permet davantage de saisir les logiques de reproduction que celles de changement; les notions de traditions, et de Culture s'avèrent trop « consistantes » et figées ; et l'approche en termes de réseaux et de ressources, si elle permet de bien décrire les parcours et leurs tournants, peine à offrir une lecture satisfaisante des phénomènes de recompositions identitaires à l'œuvre.

\section{Récits et description dense}

La familiarité construite avec quelques uns des membres des familles suivies nous a donné accès à des tranches de vie parfois difficiles. Les événements douloureux exposés ne doivent toutefois pas amener à conclure que les Turcs sont particulièrement 《 frustes $»^{7}$ et ont des itinéraires beaucoup plus chaotiques que d'autres populations. La nature des événements relatés là tient à la démarche suivie, la connaissance sur une durée suffisamment longue de personnes avec lesquelles s'établit une certaine confiance donne accès à des dimensions de leur vie qu'un récit ponctuel laisse rarement filtrer. Une histoire recueillie et racontée après coup dans un exercice où il s'agit de rendre compte de sa vie n'a pas la même tonalité et est plus "lissée », selon le terme de Pierre Bourdieu (Bourdieu, 1986), que la réaction à chaud à des événements qui viennent de se passer. Ainsi les chroniques reconstituées, dont l'extrait présenté ici, font-elles fortement écho à celles proposées par Oscar Lewis (1961) ou Artun Unsal (1989). L'écriture anthropologique remplit alors une fonction descriptive essentielle et conduit vers ce que Clifford Geertz appelle thick description (1986) : Une description en profondeur de l'objet qui prend en compte ses configurations internes de rapports sociaux, ses rapports de pouvoir, ses tensions, ses processus de reproduction permanente, ses dynamiques de transformation. Dans notre cas, cette interprétation descriptive a tenté de suivre un double cheminement : celui des migrants et celui du chercheur, liant récits de la migration et récits des situations de terrain, étapes des trajectoires et étapes des élaborations théoriques. Effacer les épreuves du terrain et le travail d'élaboration progressif de la compréhension et de l'analyse (ce que Bourdieu nomme en référence à Joyce "work in progress ») c'eût été effacer le sens même de ce qui était décrit.

\section{Histoires de famille}

La famille SATICI, originaire de la région du centre ouest de la Turquie est installée dans la Loire depuis 1973. Il s'agit en fait de deux grandes familles, déjà liées par des alliances à la génération précédant celle des migrants. Nous avons été en contact avec eux par le biais d'Elif et Hanife, deux adolescentes membres d'un groupe folklorique

7 Ce terme a été utilisé dans certains articles publiés par Gaye Salom, responsable de l'association ELELE qui a œuvré à la reconnaissance de la « problématique » turque en France. 
turc. Elles vivent avec leur mère Emine, qui a divorcé de son mari Torun depuis quelques années alors qu'il entendait prendre une autre épouse — pratique que d'autres hommes de cette importante famille ont eût également. Elles ont eu un frère, Mustafa, ainsi qu'une plus jeune sœur. Le groupe familial dont ils font partie est riche de nombreux frères et fils et bien implanté dans des activités commerçantes et entrepreneuriales au moment où nous les rencontrons ${ }^{8}$. Emine, la mère, ne porte pas le voile et les filles jouissent d'une certaine liberté : loisirs sportifs et fréquentation des copains du folklore en dehors du cadre familial. Alors qu'elles ont 19 et 20 ans lors de notre première rencontre, il n'est pas encore question de mariage. Nous avons entretenu avec cette famille, et notamment Elif, la fille aînée, une relation quasi amicale pendant cinq ans. Il n'est pas possible de consigner ici tous les évènements survenus dans ce laps de temps, ainsi nous situerons rapidement les situations de Elif et Hanife, pour exposer plus précisément les évènements qui concernent Mustafa. Cette chronique se déroule entre la France et la Turquie, entre St-Étienne et d'autres communes de Loire et de Haute-Loire, entre le salon familial et la prison. Elle mêle les registres de l'amour et de l'honneur, ceux de la violence et de la négociation, ceux de l'identité et des intérêts matériels. Elle met enfin en scène divers usages de la parenté comme valeur et comme réseau et ainsi les réinterprétations, combinaisons, recompositions dont elle est l'objet dans le contexte migratoire. Nous verrons alors comment ces intrications imposent à l'anthropologue de réviser sa boite à outils conceptuels.

Lors d'un premier épisode, Elif avait accepté la demande en mariage d'un cousin au cours d'un séjour en Turquie. Une semaine après la célébration, elle a rompu ses fiançailles, provoquant des violences à son encontre. C'est finalement une réinterprétation de son refus dans le registre de la magie et des sorts qui avait permis de trouver une issue à la situation 9 . La mobilisation de ce registre par cette famille venait remettre radicalement en cause la perception que nous en avions et par là même les dichotomies classiques entre logiques «traditionnelles » et aspirations « modernes »; la magie venant ici au service de la liberté de choix conjugal. Par la suite Elif a épousé un homme, fonctionnaire de police résidant à Istanbul, rencontré par le biais de connaissances communes. Elle l'a choisi librement et leur relation a été essentiellement nourrie par une correspondance épistolaire. Le père de Elif, alors en Turquie auprès de sa seconde femme, a été chargé de faire les démarches rituelles de demande en mariage. Son mari l'a rejointe quelques mois plus tard en France, un peu malgré lui. Sa situation professionnelle s'en trouve de fait nettement affectée : il travaille sur des chantiers grâce à sa belle famille dont il est dès lors totalement tributaire. Elif travaille comme secrétaire et ils ont un appartement indépendant.

Sa sœur Hanife, après avoir fréquenté ouvertement Yusuf, un copain du groupe folklorique dont elle faisait partie, l'a épousé lors d'une cérémonie traditionnelle, mais sans faire de mariage administratif. Ils se sont installés dans un appartement de l'im-

$8 \mathrm{Ce}$ cas de figure concerne aussi un autre grand groupe familial rencontré en France. Nous avons analysé comment la présence en nombre de membres masculins issus d'une même fratrie dès le début de l'immigration contribue à une réussite significative dans l'entrepreneuriat (commerce de fruits et légumes, épicerie, vêtements, import-export de tapis et de meubles, bijouterie pour les uns, maçonnerie, boulangerie industrielle pour les autres). Dans le second groupe familial se trouvent également plusieurs situations de bigamie, incluant une union mixte.

9 Pour un récit détaillé de ces évènements voir Autant-Dorier (2004). 
meuble vétuste appartenant à ses beaux-parents. Hanife subit le contrôle permanent de sa belle-mère et a dû interrompre ses études. Yusuf travaille dans le bâtiment avec son oncle. Le caractère librement consenti de leur union, comme le fait qu'ils soient tous les deux nés et aient grandi ici, ne constituent pas, semble-t-il, des conditions suffisantes d'autonomisation vis-à-vis de l'emprise familiale. La précarité économique renforce clairement la nécessité de recourir au réseau familial. Il faudra attendre que son mari surprenne une scène d'insultes pour qu'il fasse cesser cette emprise. Il commence alors à acquérir du matériel pour travailler à son compte.

Mustafa a séjourné en Turquie pendant une année, envoyé par sa mère pour le tenir à distance des tentations délictueuses qu'il avait en France. Il a vécu avec son père dans la période où celui-ci y était, puis auprès de sa famille maternelle. La mobilité, au delà d'un retour simple au lieu d'origine, apparaît ici comme une possibilité toujours actualisable pour faire face à des difficultés comme pour saisir des opportunités (Appaduraï, 2001).

De retour en France, Mustafa travaille dans le bâtiment avec Yusuf, son beau frère. Il rencontre Nurcan quelques mois plus tard.

\section{Carnet de terrain, St Étienne ${ }^{10}$ Récit du mariage de Mustafa et Nurcan}

Nurcan habitait dans une commune semi-rurale, au nord de Saint-Étienne, là où vit une grande partie de la famille SATICI, les deux familles se connaissent d'ailleurs bien. Mustafa a fait la connaissance de Nurcan aux fiançailles de sa sœur, Hanife. Pendant deux mois ils se sont vus ponctuellement, puis Nurcan a été surprise par son oncle en compagnie de Mustafa. Ceci a provoqué des remous et Nurcan a fait comprendre à Mustafa qu'il devait franchir le pas et être prêt à officialiser les choses. Cependant la demande directe en mariage ne semblait plus possible puisque il y avait déjà eu un affront à l'honneur familial. Nurcan a donc décidé de fuguer avec Mustafa qui était d'accord, ainsi que sa mère. Il est donc venu « l'enlever » à deux heures du matin [l'enlèvement est une pratique admise, visant à imposer le mariage aux parents de la jeune fille]. Elle est partie avec un minimum d'affaires et ils sont allés se cacher en Haute-Loire, auprès de la tante d'Emine SATICI (mère de Mustafa). Leur « retraite » a duré presque un mois.

Pendant ce temps la famille de Nurcan suppliait Emine de révéler le lieu où se cachait leur fille. Emine entamait des négociations pour que ceux-ci acceptent le mariage. De fait, la famille SATICI, en particulier le père de Mustafa n'a pas bonne réputation sur le plan moral, ainsi la famille de Nurcan ne voulait pas la leur donner, c'est d'ailleurs pour cela que Nurcan avait préféré fuir selon Elif. Le père de Nurcan a

10 Dans une première étape du travail de terrain des entretiens plus formels ont été conduits et enregistrés. Par la suite, visites amicales et recueils de données se sont confondus rendant l'usage d'un magnétophone incongru. C'est donc au travers de notes de terrain détaillées que les histoires ont été reconstituées. Elles mêlent ainsi les faits relatés, les explications et justifications qui me sont données à ce moment là et mes premières interprétations. C'est également un choix d'écriture que de les présenter comme telles, avec les incertitudes et maladresses qu'elles laissent transparaître (NB : Les éléments de récit concernant d'autres membres de la famille que Mustafa ont été coupés ici.). 
|posé comme condition initiale à toute réelle négociation que ce soit un oncle en particulier, du côté de la mère de Mustafa qui vienne faire la demande : il était l'un de ses meilleurs amis dans les premières années de la migration. Celui-ci a accepté d'intercéder en faveur de Mustafa et Nurcan. Le mariage traditionnel a donc eut lieu, mais, comme celui de Hanife il n'est pas officialisé administrativement.

Un an plus tard...

\section{Carnet de Terrain, Saint-Étienne 30 août}

J'appelle chez Emine SATICI : c'est Nurcan, la belle-fille, qui décroche et me dit qu'elle a eu une petite fille et qu'Elif vient d'accoucher (19 août) d'un petit garçon. Hanife et Yusuf attendent une fille pour le mois d'octobre. J'appelle ensuite Elif chez elle, qui est très contente de m'entendre. Je lui propose de passer la voir. Elle m'explique que Mustafa est très fier de la ressemblance de sa fille avec lui. Il a arrêté son travail au noir dans la maçonnerie pour se mettre à son compte sur les marchés dans la vente de vêtements [C'est l'un des domaines d'activités de sa famille paternelle]. Sa mère qui avait aidé son mari dans cette activité lui donne un coup de main. Il a commencé en janvier, les affaires n'ont pas bien démarré à cause du froid, mais cela s'améliore depuis le printemps. Il a pris un peu de repos cet été et reprendra cette activité à la rentrée.

\section{8 octobre}

J'avais appelé chez Emine, je demande si Hanife a accouché, et quand je pourrais venir les voir. Hanife a accouché le 23 septembre. Emine me dit qu'Elif et son mari habitent pour l'instant chez elle car ils font des travaux chez eux. Emine me dit de venir chez elle tout à l'heure. J'arrive vers 14h30. Rapidement Elif me met au courant des dernières histoires de la famille :

La femme de Mustafa, Nurcan est partie avec sa fille (de 8 mois), quittant son mari pour retourner chez ses parents. Elif en attribue la cause aux problèmes antérieurs qui ont eu lieu entre les deux familles. Elle m'apprend à cette occasion que sa cousine était mariée avec le frère de Nurcan (figure 1). Mais ce mariage a très vite été rompu. Auparavant le père de Nurcan avait emprunté 3000 euros à Akhan, l'un des oncles paternel d'Elif, " comme on fait toujours entre Turcs ». Oncle qui se trouve à l'heure actuelle en prison (à cause des évènements relatés ci-dessous). Ainsi lorsque la rupture du mariage a eu lieu, environ un an après le prêt, Akhan a demandé qu'on lui rembourse les 3000 euros. Le père de Nurcan aurait alors nié avoir eu ce prêt.

Lorsque cette histoire avait éclaté Nurcan avait quitté une première fois Mustafa, prenant parti pour sa famille, mais elle était finalement revenue. Elle a encore tenté de partir une seconde fois. On peut supposer que la famille d'Elif a fait pression sur Nurcan pour que son père rembourse sa dette, et que c'est cela qui l'a poussée à partir, mais Elif raconte sa version des faits...

Les multiples liens offrant tous les ingrédients du problème. 
Figure 1

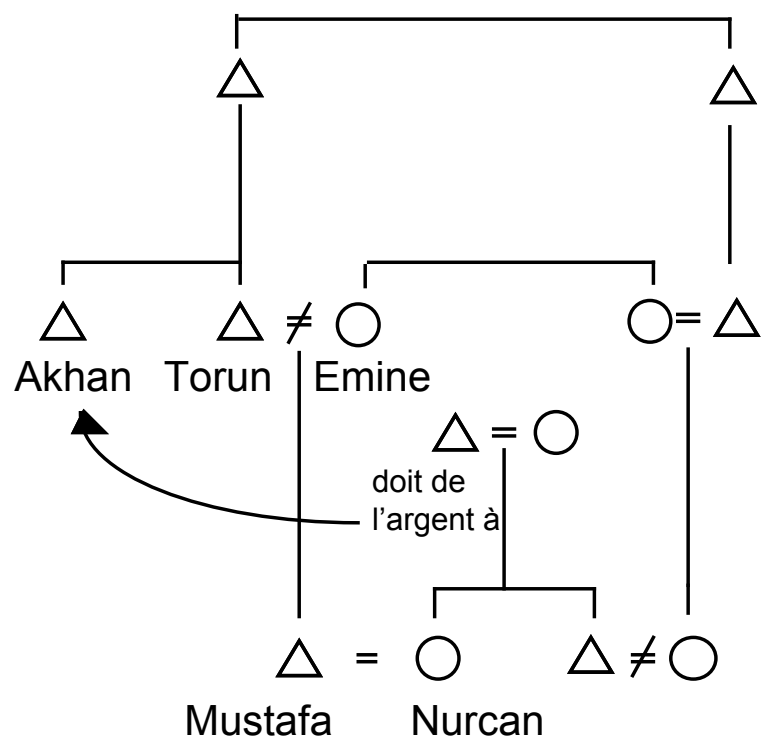

Nurcan a demandé à Mustafa de l'emmener chez ses parents. Une fois arrivée là-bas et installée dans la maison, la belle-sœur de Nurcan a pris la petite dans ses bras et l'a emmenée dans une pièce où elle s'est enfermée. Mustafa réagit en demandant qu'est-ce qui se trame. Nurcan lui aurait alors dit de partir, qu'elle ne veut plus de lui et l'aurait insulté (toujours selon Elif bien sûr). Mustafa était prêt à donner quelques coups, notamment à sa belle-mère mais il se serait retenu, celle-ci étant malade, et pensant que les conséquences pourraient être fâcheuses.

Énervé il revient chez sa mère et raconte ce qui vient de se passer, Yusuf (le mari d'Hanife, sœur de Mustafa) est lui aussi présent. Les esprits s'échauffent et Mustafa repart récupérer sa fille. Emine et Yusuf partent à sa suite. Lorsqu'ils arrivent Mustafa est en train de subir les coups et morsures (ça ne s'invente pas) de sa femme et de la famille de celle-ci.

Là le déroulement des faits devient difficile à déterminer précisément étant donné la façon passionnée dont Elif raconte comme toujours les événements : Les oncles de Mustafa, qui habitent sur place, et notamment Akhan ont été prévenus de ce qui se passait (par Mustafa avant qu'il n'arrive, ou par Emine quand elle a vu ce qui se passait ?). La bagarre prend alors de l'ampleur et la gendarmerie intervient (appelée par des voisins ou par la famille de Nurcan lorsqu'ils ont vu arriver les oncles SATICI ?). À leur arrivée Akhan s'enfuit avec sa BMW noire pour leur échapper. Il a déjà eu affaire à la police pour diverses raisons dont Elif me dit « je ne peux pas t'en dire plus » et préfère donc s'esquiver. Dans sa fuite il jette une arme (un «péteux » selon le terme utilisé par Elif) qu'il avait dans la voiture pour ne pas risquer d'être pris avec, mais un témoin, une voisine désignée comme sale raciste, l'aperçoit et décrit aux gendarmes la voiture du fuyard et où l'arme a été jetée. Les gendarmes arrêtent Akhan et enregistrent la plainte de Nurcan.

En racontant cela Elif est très virulente contre sa belle-sœur, « vipère » qui 
a toujours caché son jeu en étant souriante et gentille avec sa belle-mère, Emine, et ses belles-sœurs. Elif la traite de « horozpo » (pute, chienne). Elif et sa mère disent ne pas comprendre ce coup en traître, d'autant plus qu'Emine pour ne pas envenimer les relations avec sa belle-fille avait pris le parti de ne plus voir sa sœur (qui est aussi la mère de l'ex-femme du frère de Nurcan). Je demande si Nurcan avait fait sentir qu'elle n'était pas heureuse, si elle avait souhaité vivre ailleurs que chez sa belle-mère. On me répond que non, que lors de sa dernière rupture Emine lui avait dit qu'elle pouvait s'installer indépendamment si elle le voulait, Nurcan avait refusé. L'explication donnée est celle de la fidélité à sa famille contre celle de son mari.

Un autre problème a semble-t-il pesé sur la relation entre Mustafa et Nurcan, et a sans doute envenimé la bagarre qui a eu lieu : la question du baslik (le prix de la mariée). Le père de Nurcan avait exigé une somme de 1500 euros pour accepter de laisser sa fille se marier avec Mustafa qui l'avait enlevée. Les négociations avaient été très délicates comme nous l'avons vu, et le père de Nurcan avait voulu profiter de la situation face à cette famille connue localement pour son aisance financière. Du coup, suite au retour de Nurcan chez ses parents et après la bagarre qui a suivi, la famille SATICI a accusé le père de Nurcan d'utiliser sa fille pour se faire de l'argent, de vendre ses filles et de les reprendre! Argument tout à fait grave qui vise à attaquer l'honneur de celui-ci. De ce fait, le calcul de ce que le père de Nurcan doit à la famille SATICI est passé à 4500 euros !

Depuis la bagarre Akhan est en prison, mais son arme n'étant pas chargée il risque moins. En revanche à la demande des gendarmes de savoir ce qu'il aurait fait si elle avait été chargée il aurait répondu, sous le coup de la colère, qu'il aurait tué tout le monde. Ce qui n'arrange guère sa situation. Les gendarmes ont noté que le père de Nurcan et son frère avaient des bâtons lors de l'altercation et qu'il y avait un couteau de combat chez eux, ce qui décharge en partie les responsabilités de la famille SATICI.

Par ailleurs les autres oncles SATICI ont ordonné à Mustafa de ne plus aller là-bas. Eux en revanche ont cherché à faire pression sur la famille de Nurcan, les menaçant très explicitement. L'argument avancé, et repris avec beaucoup de conviction par Elif, est que cette petite famille de deux hommes n'auraient jamais dû chercher des noises à leur grand lignage puissant économiquement et connu pour sa manière forte de gérer les problèmes. Ils n'auraient pas dû jouer avec eux. Ainsi l'enjeu du conflit change : il n'est plus celui de l'affront de Nurcan à son mari, mais le respect du statut et de l'honneur de la famille SATICI et sa volonté d'affirmer sa domination.

Cette puissance lignagère a d'ailleurs été se faire entendre comme telle à la gendarmerie. Hier, tous les hommes de la famille présents dans la région (dont deux cousins de Belgique présents à Lyon à ce moment là) sont « descendus » à la gendarmerie, ce qui faisait au total une trentaine de voitures selon Elif (peut-être une quinzaine tout de même), ils ont demandé qu'on entende leur version des faits. Les gendarmes ont essayé de les raisonner et de faire baisser la tension, l'un d'eux aurait dit " pourquoi on se fait chier avec ces étrangers, on n'a qu'à les laisser se tuer entre eux », les autres l'ont fait taire.

Akhan risque cinq ans de prison pour port d'armes illégal et quelques autres motifs. Son « péteux » était un superbe revolver d'excellente qualité, commandé spécialement, alors qu'il possède par ailleurs des armes dont il a un permis en raison de ses locaux commerciaux et des cambriolages subis.

Elif me dit que la famille est prête à venger Akhan, son frère Mustafa se déclare 
d'ailleurs prêt à le faire lui-même puisqu'il est directement à l'origine du conflit. J'avais beau souligner les risques d'un tel choix (dans la perspective de Mustafa de récupérer sa fille cette attitude paraissait aberrante), Elif avait l'air de trouver évident qu'il n'y ait plus d'autres solutions, au mieux évoquait-elle la possibilité que ses oncles payent un arabe pour faire le « boulot»!

Pendant que je suis chez elle, Emine téléphone à la femme d'Akhan pour savoir où les choses en sont. Elle apprend alors qu'Akhan doit sortir dans l'aprèsmidi. Le coup de fil dure un long moment, puis elle vient nous expliquer les détails. Nurcan serait allée retirer sa plainte à la gendarmerie, et expliquer qu'une partie des problèmes venaient d'elle et de sa famille, qu'elle avait menti au début en disant que c'était son mari qui l'avait quittée et qu'il menaçait sa fille, etc. Ce serait la mère de Nurcan, inquiétée par les menaces pesant sur son mari et son fils qui aurait incité Nurcan à avouer la « vérité » et à désamorcer la crise. Les gendarmes fort surpris de ces aveux auraient fait comprendre à Nurcan les conséquences qu'auraient pu causer ses mensonges. L'un d'eux aurait dit que tout cela relève de la psychiatrie. Akhan est libéré mais sous surveillance. On lui interdit de venir aux abords de la maison de Nurcan et on lui garantit de payer le prix fort si jamais il est impliqué dans une nouvelle affaire.

Elif et Emine se réjouissent de cette issue favorable, où la « justice » a renforcé la puissance familiale... Quant à moi je me réjouis que cette décision permette d'éviter les projets de vengeance. Mais même après ces aveux et la libération d'Akhan l'issue ne semble pas encore évidente : la famille veut récupérer l'argent dû et montrer qu'on ne s'en prend pas à elle impunément.

À l'instar du policier, on aurait aisément tendance à l'écoute de ces récits à faire l'hypothèse de la folie. À y regarder de plus prêt d'ailleurs il y a bien de la folie dans la place : les normes mobilisées paraissent en décalage avec le cadre dans lequel elles se déploient. Plus précisément, se juxtaposent et se combinent des principes et registres d'actions qui semblent incompatibles et contradictoires. Comment en faire alors l'interprétation?

Les « histoires de couple » de ces jeunes se mêlent aux logiques de solidarité et au maillage serré du réseau de parents et d'alliés. Bien qu'une souplesse plus grande se soit manifestée pour cette famille dans la gestion du choix du conjoint, on constate que les relations matrimoniales engagent des lignages au-delà des individus qui contractent l'alliance. Mustafa est amené à s'inscrire comme membre du groupe au-delà de sa relation directe avec sa femme.

Un premier niveau d'analyse semble pouvoir rendre compte de ces situations, notamment en termes d'honneur et d'affirmation identitaire. Suzie Guth (1986 : 53) montre ainsi que « Dans un groupe à structure parentale, l'affirmation de soi est une affirmation du lien biologique, de son groupe primaire, de sa première matrice socioculturelle. Il s'agit de défendre son être le plus profond, le plus enraciné. Il faut donc mobiliser non seulement l'affectivité la plus primitive de l'ego, mais aussi les solidarités internes du groupe, le sentiment de l'honneur qu'il faut défendre, ce que Jakiez Hélias appelle le « cheval d'orgueil ». (...) Avoir de l'orgueil consiste à assumer les valeurs du groupe, à le représenter, et à en être le porte-parole. Cet apprentissage de l'orgueil va de pair avec l'apprentissage de la soumission, des interdits, ainsi qu'avec l'apprentissage de la honte, 
de la réserve, de la pudeur. (...). »

Mais « l'affirmation de soi » est-elle réductible à la seule « affirmation du lien biologique, de son groupe primaire »? Et l'honneur constitue-t-il vraiment la valeur essentielle et incontournable dans le contexte étudié ? Dans certains moments de l'action sans doute, dans le récit épique qui en est fait certainement, mais il parait douteux que cela soit toujours le cas pour Mustafa ou dans cette famille d'une façon générale. La mobilisation de « l'honneur » apparaît davantage comme une mise en scène à laquelle sont convoquées les autorités légales. Jouant avec les normes du droit et celles de la tradition, mais aussi avec les représentations que les autres s'en font (notamment le policier prêt à les laisser s'entretuer), cette utilisation permet de dépasser les unes et les autres ${ }^{11}$.

Dans ces situations, la multiplicité des normes à l'œuvre, les combinaisons et réorientations auxquelles cela donne lieu, comme les différents registres d'interprétation que les acteurs mobilisent après coup pour en rendre compte rendent inopérantes les interprétations en termes d'intégration ou de repli comme celle en termes de Culture (acculturation, déculturation). C'est ici la fluidité des référents et des identités qui paraît plutôt se manifester (Tarrius, 1999 ; Missaoui, 2003). Utiliser ces normes et registres fait écho à ce que Hymes et Gumperz (1989) ont désigné comme switching code, c'est-à-dire la capacité à changer et à combiner des codes divers selon le contexte. La multiplicité des référents n'apparaît de ce point de vue comme un dysfonctionnement que si l'on considère ces codes comme des valeurs absolues, or ils ne font sens qu'en situation. La mobilisation du registre de la magie ou de celui de l'honneur relèverait donc ici davantage d'un acte de métissage ${ }^{12}$ " qui transforme, métamorphose et rend méconnaissable ce qui était » plutôt qu'il ne juxtapose des apports clairement identifiables (Laplantine et Nouss, 1997). L'enjeu est de comprendre alors comment les combinaisons s'opèrent et se cristallisent et de sortir des conceptions classiques qui évoquent plutôt le tiraillement entre diverses appartenances, entre tradition et modernité, entre détermination et liberté...

Dans cette optique, Jean-Loup Amselle (2001) propose de substituer la notion de branchement à celle de métissage. La métaphore du branchement, moins biologique que celle du métissage, conduit à focaliser l'attention sur les opérateurs de branchements et sur les flux, sur les objets et significations culturelles qui circulent plutôt que sur la Culture. Complémentaire de la notion de branchements, celle d'attachements, proposée par Bruno Latour, permet de faire nous semble-t-il un pas de plus (2000). Considérer les attache-

11 La scène de la démonstration de la puissance familiale jusque dans le commissariat de police n'est pas sans évoquer, dans une proportion certes plus modeste, celle décrite par Lamia Missaoui (2003) à propos des gitans. Ils mobilisent à travers toute la région sud-ouest et au-delà le réseau familial et ethnique afin de faire pression sur un hôpital prestigieux qui refuse d'opérer un de leurs enfants en priorité. La mobilisation de plusieurs dizaines de personnes permet à un groupe qui n'a habituellement pas voix au chapitre d'imposer un traitement du problème qui lui est favorable. Alors qu'ils sont habituellement stigmatisés, ils jouent de leur stigmate (la violence supposée des Gitans, ou ici des Turcs) et des ressources spécifiques dont ils disposent (la capacité à mobiliser un grand nombre de personnes liées par des caractéristiques ethniques ou des réseaux lignagers) pour obtenir ce que même les non-stigmatisés ne pourraient avoir.

12 Laplantine et Nouss (1997) précisent que « le métissage n'est pas un état ou une qualité il est de l'ordre de l'acte». 
ments c'est examiner précisément comment sont reliés dans des réseaux socio-techniques (culturels, pourrions nous ajouter), les personnes, les objets, les représentations, les savoirs et savoir-faire, sans prédéfinir ce (ceux) qui opère les branchements ${ }^{13}$.

Ainsi, plutôt que de faire l'hypothèse d'une affirmation identitaire renvoyant à ce qui serait une Culture turque de l'honneur, peut-on identifier dans l'histoire de Mustafa comment les différends financiers, combinés à des attachements affectifs et matrimoniaux, conduisent les acteurs à mobiliser leur attachement à la parenté et à l'honneur ; mais aussi à faire appel aux autorités locales pour légitimer le recours à la violence et affirmer leur position dans un contexte local de solidarité et de concurrence qui attachent entre elles des familles en migration.

Les branchements qui s'effectuent font circuler des référents — des faitiches pour Latour ${ }^{14}$ - qui sont re-signifiés. Mais les individus sont pris dans ces branchements tout autant qu'ils les actualisent. La parenté (comme liens de sang et d'alliance concret et aussi comme mode de relation valorisé symboliquement), les affaires (dans leur dimension pragmatique et dans les spéculations et coups de chance qu'elles supposent), les circulations (comme déplacements tangibles et au travers de leur portée culturelle moins immédiatement saisissable), la magie et l'honneur, la nationalité française (prise collectivement par les membres de la famille et qui facilite les circulations pour affaires vers la Turquie et en Europe) sont ainsi autant de faitiches qui font faire les acteurs ${ }^{15}$.

Ce point de vue permet dès lors de décrire comment les identités se font élaborer - plutôt qu'elles ne sont élaborées ou qu'elles ne s'élaborent — dans un mouvement incessant de circulation. Suivre la chaine des attachements aux faitiches au travers des actes et du sens qui leur est conféré conduit à dessiner un réseau de significations intelligible malgré les apparentes contradictions. Réseau de significations auquel le chercheur contribue : les récits et justifications données à l'ethnologue sont autant de branchements effectués en réponse à une jeune femme française, qui elle-même les interprétera. Notons

13 Il n'est sans doute pas insignifiant que Latour, comme Amselle, mais c'est aussi le cas de Hannerz (1997), en viennent à proposer ces outils après avoir eu une approche en termes de réseau (réseaux marchands africains, réseaux de la science, réseaux sociaux urbains). Ils permettent de sortir des conceptions essentialistes et ouvrent à une analyse dynamique des connexions plutôt qu'à la réification d'entités déjà là.

14 Ce néologisme mêle les notions de « fait » et de « fétiche », le fait représentant quelque chose de vérifiable, dans lequel on peut avoir confiance, le rationnel dont on est sûr ; et le fétiche, renvoyant à quelque chose d'irrationnel, de symbolique, auquel on croit. Le faitiche est ce à quoi l'on est attaché, et est dans le même temps ce que l'action fabrique. Les deux mots de fait et fétiche sont tous deux rapportés à l'étymologie du verbe faire (les faits sont faits par la science et le fétiche fait faire). Bruno Latour incite par là à considérer plus précisément l'action avec l'idée de « faire faire », qui permet de se pencher davantage sur ce qui fait agir : "Avec cette formule on ne cherche plus à répartir ce qui fait et ce qui est fait, on parcourt une chaine de médiateurs dont aucun ne cause exactement le suivant mais dont chacun permet à son tour de devenir origine de l'action ». (Latour, $2000: 197$ ).

$15 \mathrm{Au}$ nombre de ces faitiches il conviendrait également d'ajouter les lieux, espaces urbains repérables ou plus invisibles, marqués par les ordres locaux et reconfigurés par les passages des migrants, notamment commerçants. (Missaoui et Tarrius, 2006). 
au passage, que le partage entre l'enquêteur et l'enquêté, le chercheur et son " objet » tend ainsi à être fortement remis en cause. Le chercheur devenant un médiateur parmi d'autres.

\section{POUR CONCLURE}

C'est à un basculement de perspective qu'invitent ces considérations, réaffirmant ce que devrait être la tâche de l'anthropologue, particulièrement lorsqu'il s'attache à comprendre les mobilités. Elle consisterait à mettre en évidence la complexité des attachements et la diversité des faitiches, y compris des siens, pour éviter les catégorisations et leurs effets performatifs. Il ne s'agit cependant pas de croire qu'il n'y aurait que fluidité et communication, mais l'enjeu est d'éviter, autant que faire se peut, de conforter les grilles d'analyse normatives de l'altérité (Streiff-Fénart, 2006). Cela ouvre à d'autres chantiers de recherche anthropologique dont l'enjeu est de questionner les multiples catégories, procédures, contraintes et ressources attachées aux migrants ou à ceux considérés comme tels et ce que cela fait faire. Il s'agit notamment d'examiner les situations d'asile, la construction des identités de papiers et de sans-papiers (Ferjani, 2000 ; Belkis et Franguiadakis, 2005 ; Têtu, 2007), mais également les situations de discriminations et de mobilisations contre celles-ci, les savoir-faire, les dispositifs et les tactiques utilisées. Dans cette perspective, les formes identifiées ne sont pas systématiquement celles de la lutte et de la revendication, mais celle de la négociation et de la persévérance. Elles font émerger des figures de médiateurs (témoin, traducteur, diplomate) qui combinent reconnaissance de l'Autre et formes d'accompagnement (Autant-Dorier, 2008). Ces analyses conduisent à réinterroger les modalités de la citoyenneté : «Jusqu'à quel point les procédures, les instruments, les règles, les techniques des « milieux traducteurs » favorisent-ils une ressaisie par les institutions des problèmes rencontrés par ceux qui, dans l'affirmation d'un droit à avoir des droits, s'érigent comme des « sujets » politiques légitimes ?» (www.profacity.eu, 2008) ${ }^{16}$.

\section{Références bibliographiques}

AMSELLE Jean-Loup (2000) La globalisation. Grand partage ou mauvais cadrage ?, Intellectuels en diaspora et théories nomades, L'Homme, ${ }^{\circ} 156$, pp. 207-225.

AMSELLE Jean-Loup (2001) Branchements. Anthropologie de l'universalité des cultures, Paris, Flammarion, $265 \mathrm{p}$.

APPADURAI Arjun (2001) Après le colonialisme, les conséquences culturelles de la globalisation, Paris, Payot (édition originale 1996), 319 p.

16 C'est à ce chantier que s'attellent plusieurs collègues anthropologues dans une équipe internationale et pluridisciplinaire rassemblée pour un programme européen sur les citoyennetés profanes. "L'hypothèse centrale de Profacity est que les entreprises profanes de requalification de la citoyenneté en Europe ne sont pas à considérer seulement dans l'ordre de la validation et de l'extension des droits juridiques à de nouveaux « cas » ou à de nouvelles catégories sociales. (...) Les citoyennetés profanes expérimentent des formes de citoyenneté, portées par des sans parts et des ayant parts agissant en solidarité, qui visent à modifier les délimitations de la citoyenneté juridique. », http://www.profacity.eu/modules/news/article.php?storyid=73. 
AUTANT-DORIER Claire (2004) Traversée de frontières. L'identité combinée d'une jeune fille de France et de Turquie au fil du temps, in Claire Cossée, Emmanuelle Lada et Isabelle Rigoni Dir., Faire figure d'étranger, regards croisés sur la production de l'altérité, Armand Colin, Paris, pp. 103-118.

AUTANT-DORIER Claire (2007) La parenté « faitiche » ou Que fait-on faire à la parenté ? Des familles turques en migration, European Journal of Turkish Studies, Thematic Issue n ${ }^{\circ} 4,4$, The social practices of kinship. A comparative perspective, http://www.ejts.org/document597.htm.

AUTANT-DORIER Claire et BATTEGAY Alain (2007) Économie morale dans des parcours de migrants entre la France et l'Algérie et entre la Turquie et la France. Moments, scènes et portraits, in J.-F. Leguil-Bayard et Fariba Adelkhah Dir., Voyages du développement, Karthala, CERI, pp. 269-308.

AUTANT-DORIER Claire (2008) Des agents « entremetteurs de reconnaissance » : le traitement de l'altérité aux marges de l'institution, in J.-P. Payet et A. Battegay Coord., La reconnaissance à l'épreuve. Explorations socio-anthropologiques, Presses universitaires du Septentrion, pp. 123-128.

BELKIS Dominique et FRANGUIADAKIS Spyros (2005) Accueillir les demandeurs d'asile. Quand la relation à l'autre fabrique le politique, Écarts d'identité, n 107 , vol. II, Grenoble, pp. 13-20.

BOURDIEU Pierre (1980) Le sens pratique, Paris, Éditions de minuit, 475 p.

BOURDIEU Pierre (1986) L'illusion biographique, Actes de la Recherche en sciences sociales, $n^{\circ} 62-63$.

DE TAPIA Stéphane (2005) Migrations et diasporas turques : circulation migratoire et continuité territoriale, 1957-2004, Paris, Maisonneuve \& Larose, 402 p.

FERJANI Mohamed Chérif (2000) Identité(s) sans papiers, identité(s) de papiers, Écarts d'identité $\mathrm{n}^{\circ} 93$, pp. 2-5.

FERRIE Jean-Noël et BOETSCH Gilles (1992) L'immigration comme domaine de l'anthropologie, Cahiers de l'IREMAM, $\mathrm{n}^{\circ} 2$.

FLICHE Benoît (2007) Odyssées Turques, Les migrations d'un village anatolien, Paris, Éditions du CNRS, $236 \mathrm{p}$.

GEERTZ Clifford (1986) Savoir local, savoir global. Les lieux du savoir, Paris, PUF, 293 p.

GEERTZ Clifford (1998) La description dense. Vers une théorie interprétative de la culture, Enquête, $\mathrm{n}^{\circ} 6$.

GOKALP Altan, KASTORYANO Riva et DE TAPIA Stéphane (1997) L'immigration turque et kurde : la dynamique segmentaire, la nouvelle donne générationnelle, et le nouvel ordre communicationnel, Rapport, Appel d'offre du FAS.

GUTH Suzie (1986) Le conflit et la morphogenèse des groupes, in P. Watier Dir., Georg Simmel. La sociologie et l'expérience du monde moderne, Paris, Meridiens Klincksieck.

HANNERZ Ulf (1983) Explorer la ville. Éléments d'anthropologie urbaine, traduit de l'anglais (États-Unis) et présenté par Isaac Joseph, 432 p. (coll. Le sens commun).

HANNERZ Ulf (1997) Borders, International Social Science Journal, $\mathrm{n}^{\circ}$ 154, Anthropology Issues and perspectives: II - Souding out new possibilities, Blackwell Publishers/UNESCO, pp. 537-548.

HYMES Dell et GUMPERZ John (1989) Engager la conversation, Paris, Éditions de Minuit, 185 p.

LAPLANTINE François et NOUSS Alexis (1997) Le métissage, Paris, Flammarion, 127 p. (coll. Dominos).

LATOUR Bruno (1997) Nous n'avons jamais été modernes. Éssai d'anthropologie symétrique, La Découverte/poche, (1 ère édition, 1991), $206 \mathrm{p}$.

LATOUR Bruno (2000) Factures/fractures : de la notion de réseau à celle d'attachement, in André Micoud et Michel Peroni Éds., Ce qui nous relie, CRESAL, Éditions de l'Aube, pp. 189-206.

LEVI-STRAUSS Claude (1967) Les structures élémentaires de la parenté, Paris, Mouton, $591 \mathrm{p}$.

LEWIS Oscar (1961/trad. 1963) Les enfants de Sanchez, autobiographie d'une famille mexicaine, Gallimard, 638 p. 
MISSAOUI Lamia (2003) Les étrangers de l'intérieur. Filières, trafics et xénophobie, Paris, Payot, $272 \mathrm{p}$.

MISSAOUI Lamia et TARRIUS Alain (2006) Villes et migrants, du lieu-monde au lieu-passage, Revue Européenne des Migrations Internationales, vol. 22, n 2, pp. 43-65.

TARRIUS Alain (1995) Arabes de France dans l'économie mondiale souterraine, l'Aube, essai, $219 \mathrm{p}$.

TÊTU Marie-Thérèse (2007) L'énigme des sans papiers algériens en France. De la conversation à l'ethnographie multi sites, Anthropologie et Sociétés, Entre-lieux de l'humanitaire, n ${ }^{\circ}$, vol. 31 , Université Laval.

RICOEUR Paul (1983-1985) Temps et récits, 3 tomes, Paris, Seuil.

RIGONI Isabelle (2001) Mobilisations et enjeux des migrations de Turquie en Europe de l'Ouest, Paris, L'Harmattan, $463 \mathrm{p}$.

STREIFF-FENART Jocelyne (2006) À propos des valeurs en situation d'immigration : questions de recherche et bilan des travaux, Revue française de sociologie, 47-4, pp. 851-875.

TODD Emmanuel (1994) Le destin des immigrés. Assimilation et ségrégation dans les démocraties occidentales, Paris, Seuil, 390 p.

UNSAL Artun (1989) Chronique d'une famille anatolienne, Lettres turques, L'Harmattan, 153 p.

VIDELIER Philippe (1993) La migration comme configuration du monde, Ethnologie Française XXIII, 2.

ZONABEND Françoise (1980) La mémoire longue. Temps et Histoires au village, Paris, PUF, 314 p. 


\title{
Saisir les identités en mouvement : parenté et histoires de familles turques en migration
}

\author{
Claire AUTANT-DORIER
}

La question de l'approche anthropologique des migrations est abordée lors d'un parcours de recherche mené auprès de populations turques pendant plusieurs années. Dans la démarche de terrain, comme dans celle d'écriture, est identifié ce qui pourrait relever d'une spécificité de l'anthropologie : notamment une approche attentive au sens que les migrants donnent à leurs parcours, caractérisée par une ethnographie multilocalisée et le suivi des histoires de famille dans la durée. Le cheminement de la recherche a conduit à s'interroger sur les outils et ressources théoriques susceptibles de rendre compte des phénomènes observés. L'analyse des phénomènes migratoires apparaît de ce point de vue particulièrement propice à une remise en question des catégories de l'analyse (parenté, culture, tradition, intégration, etc.) et amène à repenser la question des appartenances. L'article examine comment s'opèrent les branchements culturels et les attachements en situation. Il défend une anthropologie des identités en mouvement, attentive à dépasser les habituels partages entre repli et intégration, tradition et modernité, mais aussi entre le chercheur et son « objet».

\section{Grasping Identities on the Hove: Parenthood and Histories of Turkish Migrant Families}

\author{
Claire AUTANT-DORIER
}

Questions arising from an anthropological approach of migrations are raised through a research process conducted amid Turkish people for several years. During field work and the writing process, what might be considered specifically relevant for anthropology has been identified: in particular, an extremely careful multi-localized ethnographical approach to the meaning which migrants give to their routes and to the follow-up of family histories in the long run. The development of the research process led to questions about the tools and theoretical resources most likely to account for the phenomena observed. From that point of view, an analysis of migratory processes appears to be particularly favourable to questioning the categories of analysis (parenthood, culture, tradition, integration, etc.) and leads to a renewed approach of the notion of belonging. The present paper analyzes the ways in which cultural linking ups and connexions in a given situation operate. It stands up for an anthropology of identities on the move, careful to go beyond the usual divisions between withdrawal and integration, tradition and modernity, but also between the researcher and his/her object. 


\title{
Captar las identidades en movimiento: parentesco e historias de familias turcas en la migración
}

\author{
Claire AUTANT-DORIER
}

\begin{abstract}
Abordamos aquí al enfoque antropológico de las migraciones a través de un recorrido de investigaciones llevadas durante varios años con poblaciones turcas. Tanto en el proceso de investigación como en el de escritura identificamos lo que puede relevar de una especificidad de la antropología como la cuidadosa atención al sentido que los migrantes dan a su recorrido, atención caracterizada por una etnografía multi-localizada y un seguimiento, a lo largo del tiempo, de la historia de las familias. La evolución de la investigación lleva a interrogarse sobre los recursos teóricos y las herramientas capaces de dar cuenta de los fenómenos que observamos. De este punto de vista el análisis de los fenómenos migratorios permite poner en tela de juicio las categorías del análisis (parentesco, cultura, tradición, integración, etc.) y lleva a replantearse la cuestión de las pertenencias. El artículo examina cómo se operan en situación los empalmes culturales y los lazos. Defiende una antropología de las identidades en movimiento, atenta a superar las oposiciones entre repliegue e integración, tradición y modernidad, y también entre el investigador y su «objeto».
\end{abstract}

